\title{
Preventing autoimmunity by regulating regulatory T-cell induction
}

\author{
Friedrich Luft
}

Published online: 26 September 2009

(C) Springer-Verlag 2009

Non-obese diabetic (NOD) mice are susceptible to spontaneously develop autoimmune insulin-dependent diabetes mellitus (IDDM). Japanese investigators developed the NOD strain almost 30 years ago by selecting cataractprone strains [1]. The NOD strain was then established by inbreeding. The susceptibility to IDDM is polygenic and the environment exerts a strong effect on gene penetrances. Environmental effects include housing conditions, health status, diet, and drugs, which all effect development of diabetes in the mice. For instance, NOD mice maintained in different laboratories can have different levels of IDDM incidence. The genes and mechanisms involved in NOD mice are not the same as in human IDDM, although as a model of IDDM to monitor target-organ damage, NOD mice do quite well. The incidence of disease is much higher if the mice are maintained in a relatively germ-free environment. Oddly, cyclophosphamide administration also potentiates their propensity to develop IDDM. Interestingly, cyclophosphamide-induced IDDM in NOD mice is associated with reduction of $\mathrm{CD} 4+, \mathrm{CD} 25+$, Foxp3+ regulatory $\mathrm{T}$ cells ([2], see below). NOD mice are also susceptible to developing other autoimmune syndromes, including autoimmune sialitis, autoimmune thyroiditis, autoimmune peripheral polyneuropathy, a systemic lupus erythematosus-like disease that develops if mice are exposed to killed mycobacterium, and prostatitis.

A substantial contribution to our understanding of autoimmunity has been the discovery of regulatory $\mathrm{T}$ cells (Tregs). Tregs are a specialized $\mathrm{T}$ cell subpopulation that acts to suppress activation of the immune system and

F. Luft $(\bowtie)$

Experimental and Clinical Research Center,

Berlin-Buch, Germany

e-mail: luft@charite.de thereby maintains immune system homeostasis and tolerance to self-antigens. Tregs express specific surface markers, CD4 and CD25, and exhibit the winged-helix forkhead box P3 (Foxp3) transcription factor. Foxp3 is absolutely essential for Treg function. Interest in regulatory $\mathrm{T}$ cells has been heightened by evidence from experimental mouse models demonstrating that the immunosuppressive potential of these cells can be harnessed therapeutically to treat autoimmune diseases, to facilitate transplantation tolerance, or to specifically eliminate potential cancer cells [3]. Suppose that instead of administering Tregs, we could easily just order the body to make more Tregs? Indeed, in this issue, Zheng et al. [4] report their experience with just that very approach. Furthermore, they also display their results in the treatment of NOD mice. Their treatment was surprisingly effective in inhibiting the development of IDDM in NOD mice.

Exactly, what are these Tregs and where do they come from? Two different signals are necessary for the activation of $\mathrm{T}$ lymphocytes and their entrance into the G1 phase of the cell cycle [5]. The first signal comes from the interaction with the antigen, and the other is a costimulatory signal. The interleukin (IL)-2 cytokine is secreted as a consequence of this stimulation. This allows the proliferation and differentiation of $\mathrm{T}$ lymphocytes into the following cellular subtypes of $\mathrm{T}$ helper (Th) lymphocytes: CD4+ T helper 1 (Th1) lymphocytes and T helper 2 (Th2) lymphocytes. Both populations of lymphocytes produce different sets of cytokines. Th1 lymphocytes primarily produce interferon IFN- $\gamma$. Th2 lymphocytes produce IL-4, IL-5, and IL-13. Th1 lymphocytes are mainly related to macrophage activation in processes of intracellular antigen elimination and in the activation of natural killer (NK) cells. NK cells induce cytotoxicity in infected cells and also produce proinflammatory cytokines as a response to an 
antigenic stimulus. Th2 lymphocytes are related to activation and differentiation of B lymphocytes. Th2 lymphocytes recognize antigens located on the surface of B lymphocytes, along with the Swiss leukocyte antigen class-II (SLA-II) molecules, and then induce the clonal expansion of B cells. As a consequence, the B lymphocytes differentiate into plasma cells, which produce antibodies. Th2 cells also participate in the activation of eosinophils. This information is complicated enough; however, we have recently been confronted with other types of Th lymphocytes (Fig. 1).

A TGF- $\beta$-dominant environment leads naïve $\mathrm{CD} 4+\mathrm{T}$ cells to commit to the Treg phenotype. This commitment is obtained by the TGF- $\beta$-induced expression of the lineageunique Foxp3. T cells can be induced to express the Treg phenotype by TGF- $\beta$; however, a natural population of Tregs also emerges from the thymus with potent regulatory properties. Humans and mice born with genetic defects in Treg production develop devastating forms of autoimmunity $[3,5]$. In addition, TGF- $\beta$, in the presence of IL- 6 or IL-21, rather than producing Tregs, promotes commitment of CD4+ T cells to the highly cytopathic Th17 phenotype. Th17 cells participate in extremely inflamed forms of $\mathrm{T}$ cell-dependent tissue injury. Th17 T cells will be the topic of a subsequent report.

Floess et al. [6] recently identified a conserved, CpGrich, Treg-specific, demethylated region in the $5^{\prime}$ untranslated region of the Foxp3 gene that showed transcriptional activity. Their data suggested that this region is an important methylation-sensitive element regulating Foxp3 expression and demonstrated that epigenetic imprinting in this region is critical for establishment of a stable Treg lineage. The investigators also reported that inhibition of DNA methylation by azacytidine, even in absence of exogenous TGF- $\beta$, not only promoted de novo induction of Foxp3 expression during priming, but also conferred stability of Foxp3 expression upon restimulation [7]. Subsequently, Lal et al. [8] identified a unique and evolutionarily conserved CpG-rich island in a Foxp3 upstream enhancer. Presumably, this site is the same methylation-sensitive element reported by Floess et al. [6]. The abbreviation $\mathrm{CpG}$ stands for cytosine and guanine separated by a phosphate (- - - phosphate $-\mathrm{G}-$ ), which links the two nucleosides together in DNA. The $\mathrm{CpG}$ notation is used to distinguish a cytosine followed by guanine from a cytosine base paired to a guanine. $\mathrm{CpG}$ islands are regions that contain abundant $\mathrm{CpG}$ sites. Methylation of $\mathrm{CpG}$ sites within genes can lead to their silencing, a feature found in a number of human cancers (Fig. 2).

Lal et al. [8] discovered that a specific site within this nonintronic upstream enhancer was unmethylated in Tregs but heavily methylated in naive $\mathrm{CD} 4+\mathrm{T}$ cells, activated CD4 $+\mathrm{T}$ cells, and peripheral TGF- $\beta$-induced Tregs. In these cells, the DNA methyltransferases DNMT1 and DNMT3 $\beta$, as well as the methyl-binding proteins MeCP2 and MBD2, bound this CpG-rich enhancer. Demethylation of this $\mathrm{CpG}$ site using the DNA methyltransferase inhibitor, 5-aza-2'-deoxycytidine (DAC), induced acetylation of histone 3 , interaction with the zinc-finger transcription

Fig. 1 After antigen activation and costimulation, naïve CD4+ T cells commit to one of four phenotypes, governed by cytokine content of the microenvironment. IL-12, IL-4, or TGF- $\beta$ commit to Th1, Th2, or Tregs, whereas TGF- $\beta$ and IL-6 fosters commitment to the Th17 phenotype

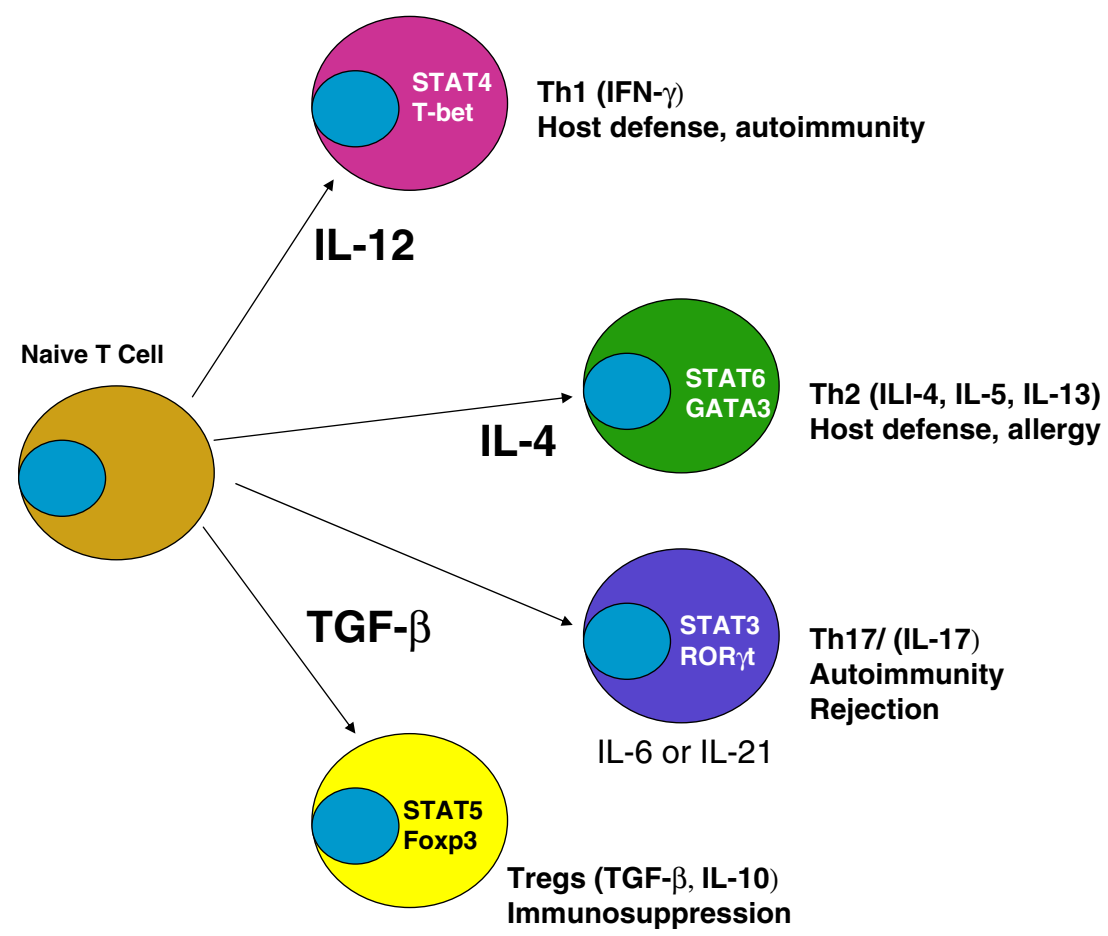




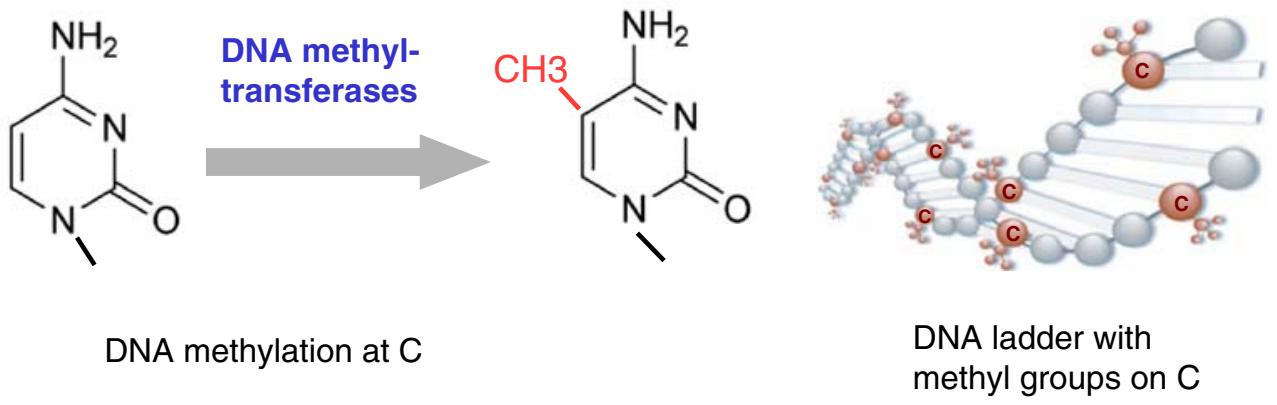

Fig. $2 \mathrm{CpG}$ sites are short stretches of DNA in which the frequency of the CG sequence is higher than other regions. It is also called the $\mathrm{CpG}$ island; a cytosine nucleotide "C" occurs next to a guanine nucleotide " $G$ " in the linear sequence of bases. "p" simply indicates that $\mathrm{C}$ and $\mathrm{G}$ are connected by a phosphodiester bond (-Cphosphate $-\mathrm{G}-$ ). DNA methylation involves the addition of a methyl

factors TIEG1 and Sp1, and resulted in strong and stable induction of Foxp3. Conversely, IL-6 resulted in methylation of this site and repression of Foxp3 expression. DAC plus TGF- $\beta$ induced CD4+, CD25+, FoxP3-expressing Tregs. Lal et al. [8] concluded that epigenetic regulation of Foxp3 could be predictably controlled with DNMT inhibitors to generate functional, stable, and specific Tregs. Moon et al. [9] treated splenic CD4+, CD25- naïve T cells from BALB/c mice with the either DAC or the histone protein deacetylase inhibitor trichostatin A (TSA). They measured the effects on Foxp3 expression. They also confirmed increased Foxp3 expression in naïve $\mathrm{T}$ cells following treatment with DAC.

Zheng et al. [4] treated C57BL6 mice with DAC and found that the treatment decreased thymus weight and thymocyte number. CD4+, CD8+, and other lymphocyte populations also decreased. Cell-cycle distribution was not affected, and apoptosis markers were decreased. However, the Treg count was decidedly increased. The authors then showed evidence that TGF- $\beta$ was involved in DACinduced Foxp3 expression. The authors also identified CD4-single-positive thymocytes and noted that CD25+ cells commonly expressed cytotoxic T-lymphocyteassociated protein (CTLA)-4, the glucocorticoid-induced tumor necrosis factor receptor family-related gene, and selectin L (CD62L). The authors also checked for $\mathrm{CpG}$ island demethylation in DAC treated mice and showed convincingly that the augmentation of Foxp3 expression was related to Foxp3 intron 1 demethylation. Zheng et al. [4] then turned their attention to the NOD mice. The authors injected the mice with cyclophosphamide at age 8 weeks to accelerate IDDM. Control mice received dimethylsulfoxide. The diabetes rate in control mice was $20 \% 2$ weeks after cyclophosphamide, which increased to $63 \%$ at 4 weeks. In contrast, no mouse receiving DAC had developed diabetes by 6 weeks. Lymphocyte infiltration and destruction of islets group to DNA - for example, to the number 5 carbon of the cytosine pyrimidine ring - in this case with the specific effect of reducing gene expression. DNA methylation at the 5 position of cytosine has been found in every vertebrate examined. In adult somatic tissues, DNA methylation typically occurs in a $\mathrm{CpG}$ dinucleotide context

were similarly reduced. Tregs were increased. The authors' data appear convincing; DAC saved the NOD mice from developing diabetes, at least in this timeframe.

There is much new information in this report for befuddled clinicians. First, we should review the process that DAC is addressing. Epigenetics is a term that describes the stable alteration of gene expression potential and encompasses both DNA methylation and histone modification. Epigenetic processes cooperate to modify either the DNA itself or the proteins that intimately associate with the DNA to alter chromatin configuration, thus establishing the characteristic gene expression profile of individual somatic tissues. In this case, the DNA is itself being modified. DNA methylation is a postreplication addition of methyl groups to cytosine residues in DNA, predominantly in the sequence CpG. DNA methylation establishes a silent chromatin state and brings about the silencing of transcription [10]. DNA methylation patterns obviously must change dramatically during embryonic development. The methylation pattern is then fine-tuned during subsequent development to yield a pattern characteristic of a particular tissue. Somatic cells in general are methylated at approximately $80 \%$ of their $\mathrm{CpG}$ sites. How $\mathrm{CpG}$ islands escape methylation under circumstances where most of the genome is methylated remains unclear. Cancer researchers have led the research into DNA methylation, since cancer cells exhibit a dramatic alteration in the distribution of methyl groups. Most tumors have a reduced frequency of $\mathrm{CpG}$ methylation. Alternatively, some $\mathrm{CpG}$ islands can become hypermethylated. As a result, genomic instability and loss of heterozygosity can occur.

Thus far, three groups of DNA methyltransferases have been identified in mammals, DNMT1, DNMT2, and DNMT3. The DNA methyltransferases induce methylation at $\mathrm{CpG}$ islands (Fig. 2). DAC results in pharmacological reduction in DNA methylation by means of DNMT inhibition. Indeed, DAC has been used as a treatment for 
myelodysplastic syndrome. However, the mechanisms of action in this and other malignancies are uncertain [11]. The pleiotropic biological effects of DNMT inhibitors and recent reports demonstrating lack of association between clinical response and methylation reversal of candidate tumor suppressor genes suggest a complex mechanism behind their clinical efficacy that could also involve a cytotoxic effect rather than solely methyltransferase inhibition.

What can DAC bring us in terms of a mechanistic tool? Tregs exert immune surveillance activities by modifying the function of antigen presenting cells, such as dendritic cells, macrophages, and B cells [12]. Tregs can induce apoptosis of such cells or inhibit their activation and function. Such Treg actions are mediated by both soluble factors (cytokines and growth factors) and cell-associated molecules, such as CTLA-4. However, in autoimmunity, chronically activated antigen presenting cells can escape surveillance by Tregs, leading to the activation of $\mathrm{T}$ cells that are refractory to suppression by Tregs. Antigen presenting cells and their derived inflammatory cytokines can render Tregs defective and can also reciprocally enhance the activity of the IL-17-producing pathogenic Th17 $\mathrm{T}$ cell subset. The information provided by Zheng et al. [4] applying DAC in an animal model could give us a convenient method to study Tregs in available models quickly. Extrapolating tools such as DAC to treating or preventing nonlethal chronic diseases in man seems far in the future. However, epigenetics as a treatment target is being actively studied in human melanoma, breast cancer, ovarian cancer, brain tumors, and in hematologic malignancies. Thus, T cells are thrilling, especially now.

Respectfully,

Friedrich C. Luft

\section{References}

1. Kikutani H, Makino S (1992) The murine autoimmune diabetes model: NOD and related strains. Adv Immunol 51:295-322

2. Brode S, Raine T, Zaccone P, Cooke A (2006) Cyclophosphamideinduced type- 1 diabetes in the NOD mouse is associated with a reduction of CD4+ CD25+ Foxp3+ regulatory T cells. J Immunol 177:6603-6612

3. Vila J, Isaacs JD (2009) Anderson AE (2009) Regulatory T cells and autoimmunity. Curr Opin Hematol 16(4):274-279

4. Zheng Q, Xu Y, Liu Y, Zhang B, Li X, Guo F, Zhao Y (2009) Induction of Foxp3 demethylation increases regulatory CD4+ $\mathrm{CD} 35+\mathrm{T}$ cells and prevents to occurrence of diabetes in mice. $\mathrm{J}$ Mol Med (this issue). doi:10.1007/s00109-009-0530-8

5. Strom TB, Koulmanda M (2009) Recently discovered T cell subsets cannot keep their commitments. J Am Soc Nephrol 20:1677-1680

6. Floess S, Freyer J, Siewert C, Baron U, Olek S, Polansky J, Schlawe K, Chang HD, Bopp T, Schmitt E, Klein-Hessling S, Serfling E, Hamann A, Huehn J (2007) Epigenetic control of the foxp3 locus in regulatory T cells. PLoS Biol 5(2):e38

7. Polansky JK, Kretschmer K, Freyer J, Floess S, Garbe A, Baron U, Olek S, Hamann A, von Boehmer H, Huehn J (2008) DNA methylation controls Foxp3 gene expression. Eur J Immunol 38 (6): 1654-1663

8. Lal G, Zhang N, van der Touw W, Ding Y, Ju W, Bottinger EP, Reid SP, Levy DE, Bromberg JS (2009) Epigenetic regulation of Foxp3 expression in regulatory $\mathrm{T}$ cells by DNA methylation. $\mathrm{J}$ Immunol 182(1):259-273

9. Moon C, Kim SH, Park KS, Choi BK, Lee HS, Park JB, Choi GS, Kwan JH, Joh JW, Kim SJ (2009) Use of epigenetic modification to induce FOXP3 expression in naïve T cells. Transplant Proc 41 (5): $1848-1854$

10. Taylor SM (2006) p53 and deregulation of DNA methylation in cancer. Cellsience Rev 2:1742-8130

11. Fandy TE (2009) Development of DNA methyltransferase inhibitors for the treatment of neoplastic diseases. Curr Med Chem 16:2075-2085

12. André S, Tough DF, Lacroix-Desmazes S, Kaveri SV, Bayry J (2009) Surveillance of antigen-presenting cells by CD4+ CD25+ regulatory $\mathrm{T}$ cells in autoimmunity: immunopathogenesis and therapeutic implications. Am J Pathol 174:1575-1587 